\title{
Role of free radicals in the pathogenesis of cystic fibrosis
}

In recent years great advances have been made in understanding the underlying genetic defect in cystic fibrosis, but the cause of the severe lung damage and pulmonary fibrosis, which are responsible for most of the deaths, remains unclear. The precise link between the cystic fibrosis gene, its biochemical consequences, and the increased susceptibility to pulmonary infection, particularly with Pseudomonas aeruginosa, has not been elucidated. It has proved to be extremely difficult to determine what constitutes a primary abnormality and what is a secondary consequence of infection. However, irregularities in ion transport leading to inspissation of mucous secretions, along with increased adherence of bacteria to epithelia and reduced mucociliary clearance, probably all contribute to the recurrent progressive pulmonary infection characteristic of the disease (fig 1). The mechanism responsible for the damage to the lungs and the agents concerned have, however, been largely ignored in the pursuit of a genetic answer to the disease. The final solution must inevitably have a genetic basis but, as this may not be realised until the turn of the century at the earliest, investigation of the physiological damage may prove to be more beneficial to those already suffering from the disease.

The role of free radicals in several diseases with pulmonary complications including hyperoxic lung injury in premature infants, ${ }^{1}$ adult respiratory distress syndrome, ${ }^{2}$ asthma, ${ }^{3}$ and the effect of environmental pollutants ${ }^{4}$ has recently attracted much attention. In all these cases it has been suggested that oxygen free radicals, produced in various ways, are implicated in the damage to the pulmonary epithelium (table 1). Evidence is now accumulating that these reactive oxygen species, in conjunction with abnormalities in protective antioxidants, may be important mediators of tissue damage in patients with cystic fibrosis,,$^{5-7}$ especially of the chronic lung injury (fig 2). This discussion is intended to provide a framework for future experimentation in the field.

\section{Nutrient antioxidant status in cystic fibrosis}

In addition to their respiratory problems, many patients with cystic fibrosis suffer from diminished pancreatic function. Blockage of the pancreatic duct by mucus leads to destruction of acinar cells in the pancreas and their gradual replacement by fibrous tissue, compromising pancreatic function. The production of pancreatic juices is decreased, leading to inadequate breakdown and absorption of fat soluble nutrients and reduced secretion of bicarbonate which is essential for creating the alkaline environment in the duodenum necessary for the activation of certain digestive enzymes. Poor absorption results in deficiencies of the fat soluble antioxidant vitamins, $\alpha$ tocopherol ${ }^{8}$ and $\beta$-carotene, ${ }^{9}$ and of other circulating components of the antioxidant system such as ferritin ${ }^{10}$ and selenium $^{11} 12$ (table 2). Ferritin is important in the chelation of iron as it prevents it acting as a catalyst in the formation of the highly reactive hydroxyl radical $\left({ }^{\circ} \mathrm{OH}\right)$, and selenium is essential for the activity of the antioxidant glutathione peroxidase.

In determining whether oxidative injury will occur it is useful to imagine a balance with oxidants on one side and antioxidants on the other. To date only one study has

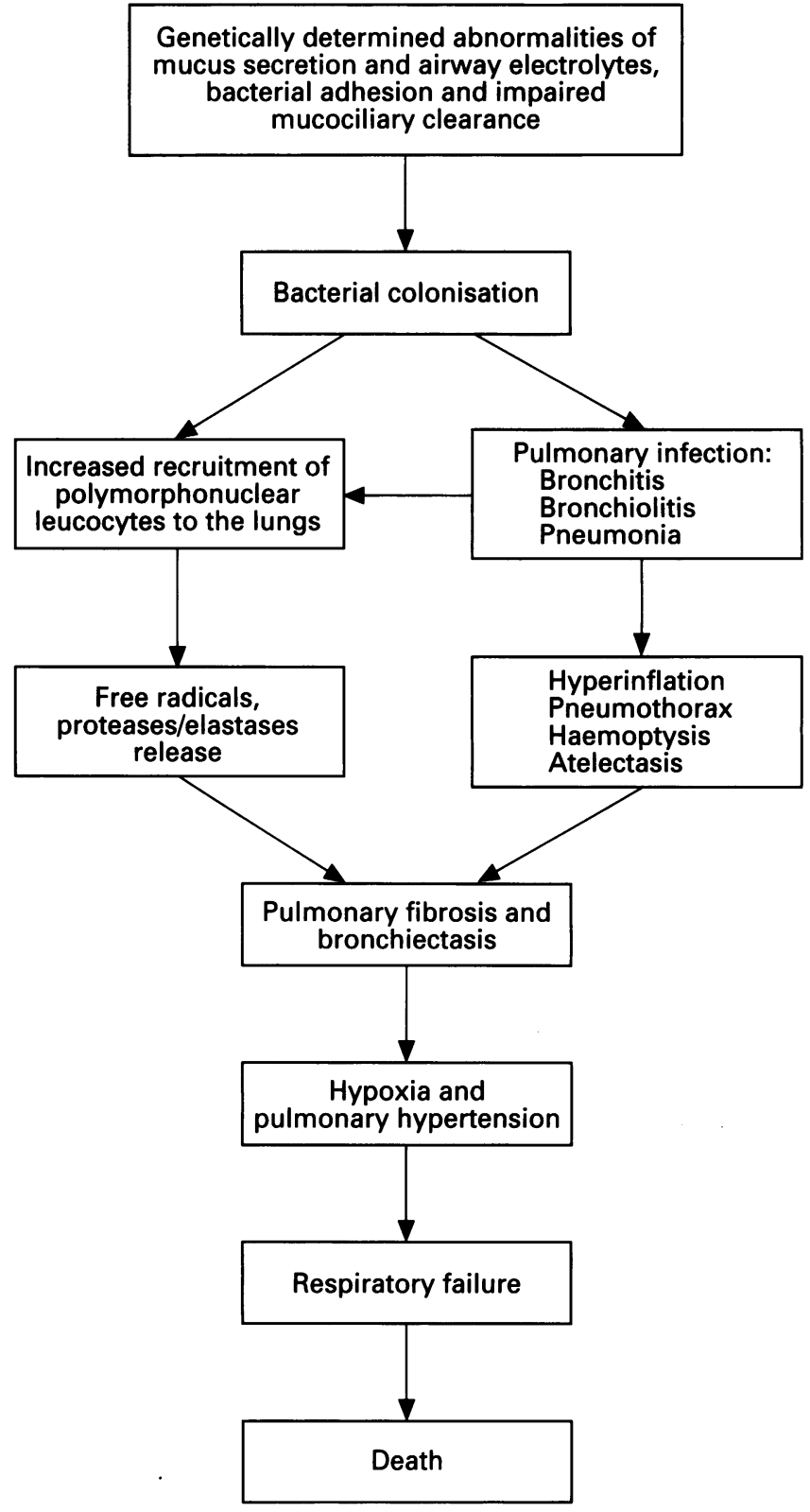

Figure 1 The natural history of cystic fibrosis in the lungs.

directly examined this balance in cystic fibrosis. Langley $e t$ $a l^{13}$ used the TRAP (total radical trapping antioxidant parameter) assay to investigate the ability of plasma from patients with cystic fibrosis to act as a buffer against lipid peroxidation initiated by an extracellular peroxyl radical generator 2,2'-azobis(2-amidinopropane) $\mathrm{HCl}$ (ABAP). They found that the buffering ability was significantly decreased when compared with plasma from age matched

Table 1 Reactive oxygen species 


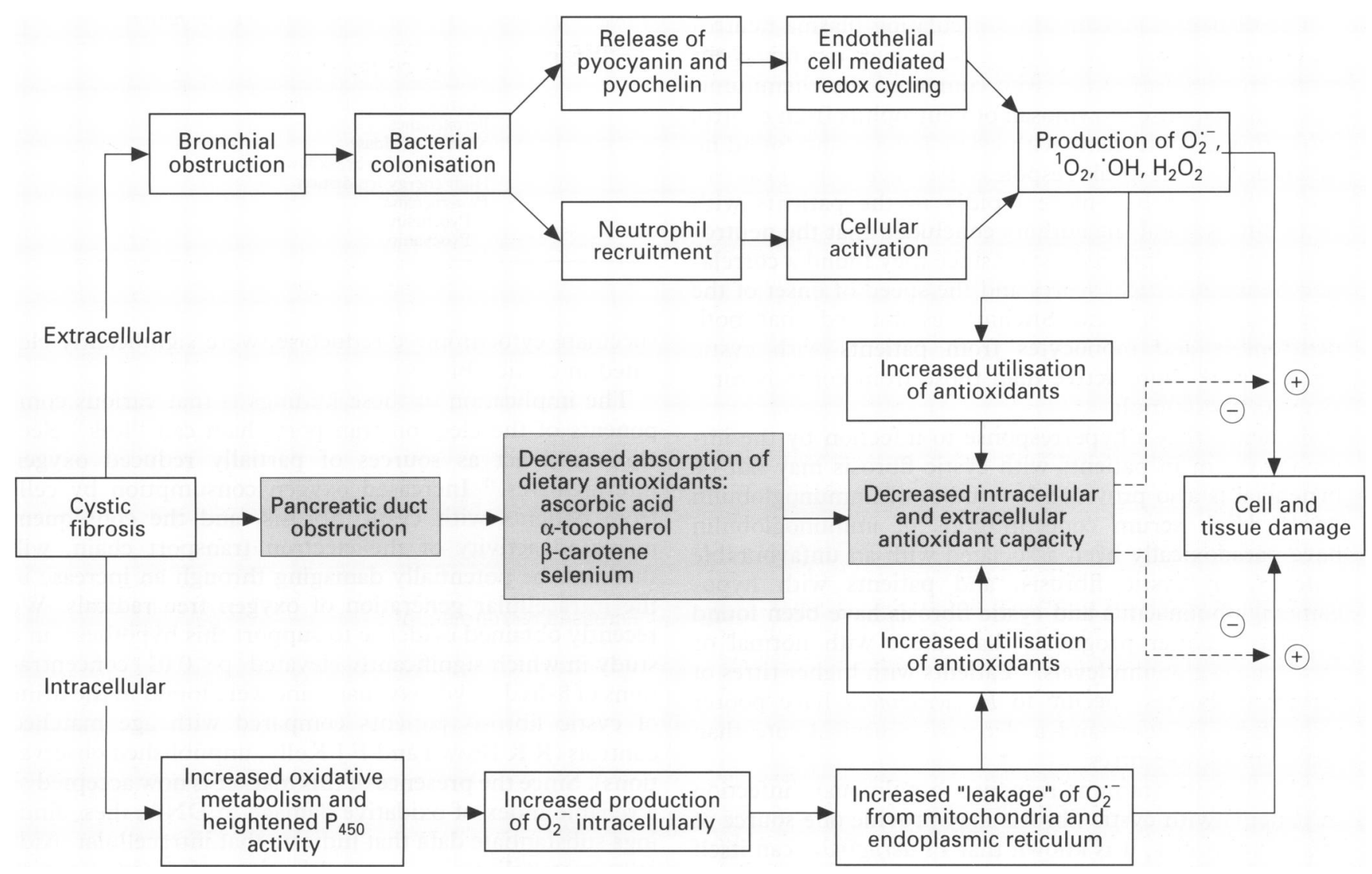

Figure 2 Proposed pathways of oxygen free radical generation and antioxidant deficiency in cystic fibrosis.

controls. They also measured the individual plasma antioxidants that contributed to the TRAP value and found that, as a consequence of modern methods of managing cystic fibrosis, plasma antioxidant defences were normal or even increased in these patients. In particular, plasma concentrations of vitamin $\mathrm{C}$ were found to be raised in a subgroup of patients with cystic fibrosis. At normal physiological concentrations vitamin $\mathrm{C}$ acts as a potent antioxidant, but at higher concentrations it has been postulated to act as a pro-oxidant in the presence of ferric iron ${ }^{14}$ by reducing it to ferrous iron which can then catalyse the formation of $\mathrm{OH}$ from $\mathrm{H}_{2} \mathrm{O}_{2}$. It has been shown in a control population that elevation of plasma concentrations of vitamin C (by oral loading) to levels equivalent to those seen in this subgroup of cystic fibrosis patients produces a similar reduction in TRAP. ${ }^{15}$ It is therefore feasible that the reduction in TRAP activity found in these patients resulted from the pro-oxidant activity of vitamin C. In their discussion Langley et $a l^{13}$ concluded that a subpopulation of children with cystic fibrosis had a reduced ability to withstand oxidative stress and therefore should, in the event of increased free radical flux, exhibit elevated indices of oxidative damage.

Table 2 Antioxidants

Intracellular
Superoxide dismutase
Catalase
Glutathione peroxidase
Glutathione
Extracellular
Vitamin E
Vitamin C
Uric acid
Sulphydryls
$\beta$-carotene

\section{Sources of oxidative stress in cystic fibrosis} INFLAMMATION

Infection of the lungs by bacteria such as Ps aeruginosa causes an extremely vigorous inflammatory response with massive neutrophil infiltration of the airways. ${ }^{16}$ Unfortunately, not only does the inflammatory response often fail to eradicate the inciting organism ${ }^{17}$ but, as a growing body of evidence suggests, it also contributes to a local defect in the host defence that interferes with eradication of the infection. ${ }^{18-20} \mathrm{~A}$ vicious cycle of infection and inflammation is therefore established. Normal homeostatic regulatory mechanisms fail to break the cycle and sustained production of inflammatory mediators continues the recruitment of additional inflammatory cells whose products cause bronchospasm, increased secretions, and other changes that exacerbate the underlying pulmonary abnormalities and lead to further deterioration in lung function. ${ }^{21}$

Alveolar macrophages are normally the predominant phagocytes obtained by bronchoalveolar lavage, with neutrophils generally accounting for less than $3 \%$ of the recovered cells. ${ }^{22}$ In contrast, in patients with cystic fibrosis, even those with mild disease, neutrophils predominate. ${ }^{23}$ Following stimulation, neutrophils and macrophages assemble a multienzyme complex that reduces molecular oxygen $\left(\mathrm{O}_{2}\right)$ to produce several highly reactive and very toxic species including superoxide $\left(\mathrm{O}_{2}{ }^{-}\right)$, hydroxyl radicals $\left({ }^{\circ} \mathrm{OH}\right)$, singlet oxygen $\left({ }^{1} \mathrm{O}_{2}\right)$, and hydrogen peroxide $\left(\mathrm{H}_{2} \mathrm{O}_{2}\right)$ (table 3). ${ }^{24}$ In the context of defence, these reactive oxygen species are considered a potent mechanism for killing organisms. At sites of inflammation oxygen radicals and proteolytic enzymes may act extracellularly and damage the host tissues. ${ }^{25}$ However, the relative importance of oxygen radicals as contributors to lung damage in cystic fibrosis is still unknown. 
The degree of activation of circulating plasma neutrophils in patients with cystic fibrosis has been examined, for example, by Graft et al ${ }^{26}$ who compared the chemiluminescence response to zymosan of neutrophils from control subjects and adolescents with cystic fibrosis and found no difference in the peak response. However, the peak response was reached more rapidly in the patients with cystic fibrosis and the authors concluded that the neutrophils were in a "primed" state since they found a correlation between clinical scores and the speed of onset of the response. Roberts and Stiehm ${ }^{27}$ established that both neutrophils and monocytes from patients with cystic fibrosis were more active than those from controls for a range of test stimuli.

Evidence that a hyperresponse to infection by the immune system in patients with cystic fibrosis may not be beneficial is also provided by studies of immunoglobulin levels. High serum concentrations of immunoglobulin have paradoxically been associated with an unfavourable outcome in cystic fibrosis, and patients with hypogammaglobulinaemia and cystic fibrosis have been found to have a better prognosis than those with normal or increased $\gamma$-globulin levels. ${ }^{28}$ Patients with higher titres of serum antibodies specific to $P s$ aeruginosa have poorer pulmonary function and a decreased survival rate than those with lower titres. ${ }^{29}$

Although the immune response to pulmonary infection in patients with cystic fibrosis may provide one source of oxidative species, it is known that Ps aeruginosa can itself produce toxic reactive oxygen species. Britigan $e t a l^{30}$ have shown that two secretory products of Ps aeruginosa pyochelin and pyocyanin - can act in a synergistic fashion to damage pulmonary artery endothelial cells in vitro. The mechanism appears to involve the endothelial cell mediated redox cycling of pyocyanin leading to the production of $\mathrm{O}_{2}{ }^{-}-/ \mathrm{H}_{2} \mathrm{O}_{2}$ which is then converted to the more cytotoxic $\mathrm{OH}$ radical through the catalytic activity of ferripyochelin. ${ }^{31}$ Additionally, Høiby and colleagues ${ }^{32}$ have shown that sputum from patients with cystic fibrosis with chronic Ps aeruginosa infection contains factors of a bacterial endotoxin and immune complex nature which are able to attract neutrophils to the lungs and to prime for enhanced release of oxygen radicals as suggested previously by Roberts and Stiehm. ${ }^{27}$ Although an increased immune response is a product of the chronic bacterial lung infection, there is now substantial evidence to indicate that it may be deleterious. Despite this, however, there is as yet very little direct evidence of oxidative damage to lung tissue to clarify the exact species involved, or the susceptibility of different lung cells.

\section{INCREASED OXIDATIVE METABOLISM}

In conjunction with the increased oxidative burden provided by the immune response to pulmonary infection in cystic fibrosis patients, there may also be an intracellular source of heightened free radical generation - namely, increased leakage from the electron transport chain in mitochondria (table 3). Feigal and Shapiro ${ }^{33}$ have shown that $\mathrm{Ca}^{2+}$ uptake and oxygen consumption is increased in the fibroblasts in patients with cystic fibrosis. They also showed that oxygen uptake was completely inhibited by cyanide, indicating that it was based on mitochondria. They used these data to hypothesise that the electron transport system in cystic fibrosis patients was more active than in controls. Von Ruecker et $a l^{34}$ expanded on this work and showed that the specific electron transfer activities of various enzymes of redox components of the respiratory chain, reduced nicotinamide adenine dinucleotide (NADH) oxidase, NADH cytochrome c reductase, and
Table 3 Potential sources of free radicals in cystic fibrosis

Activated neutrophils
Mitochondrial electron transport chain
Microsomal $\mathrm{P}_{450}$ induction
Peroxisomes
Xanthine oxidase
Catecholamine breakdown
High energy irradiation
Ps aeruginosa
Pyochelin
Pyocyanin

succinate cytochrome c reductase, were significantly elevated in cystic fibrosis.

The implication of these findings is that various components of the electron transport chain can "leak" electrons and act as sources of partially reduced oxygen intermediates. ${ }^{35}$ Increased oxygen consumption by cells from patients with cystic fibrosis, and the consequent increased activity of the electron transport chain, will therefore be potentially damaging through an increase in the intracellular generation of oxygen free radicals. We recently obtained evidence to support this hypothesis in a study in which significantly elevated $(p<0.01)$ concentrations of 8-hydroxydeoxyguanosine were found in the urine of cystic fibrosis patients compared with age matched controls (R K Brown and F J Kelly, unpublished observations). Since the presence of this marker is now accepted as a reliable index of oxidative damage to DNA, these findings substantiate data that indicate that intracellular oxidative metabolism is increased in tissues of patients with cystic fibrosis.

A further source of increased intracellular free radical generation in cystic fibrosis patients may be heightened $\mathrm{P}_{450}$ activity (table 3 ). A study of the metabolism of theophylline $\mathrm{e}^{36}$ in patients with cystic fibrosis concluded that heightened free radical production could be a reflection of enhanced $P_{450}$ activity since oxygen free radicals play an important part in oxidative detoxification reactions. These reports received further support from a study by Matkovics et $a l^{37}$ showing that the activities of intracellular antioxidant enzymes in plasma erythrocytes were elevated above control values, possibly due to "priming" by increased exposure to reactive oxygen species.

\section{Evidence for oxidative damage in cystic fibrosis}

Although the evidence for increased oxidative stress in cystic fibrosis is strong, the consequences of such stress have received relatively little attention from direct studies to determine the presence of specific markers of oxidative injury. This is partly because of technical difficulties since the only definitive way to demonstrate excessive free radical activity in vivo is by electron spin resonance study of tissues; unfortunately this cannot be used at present in clinical practice because of the nature of the spin traps. Instead investigations have to rely on the measurement of established clinical markers of free radical activity in biological fluids, most of which reflect free radical attack on polyunsaturated fatty acids in cell membranes. A classical route of free radical attack is through lipid peroxidation generating hydroperoxides, endoperoxides, long-lived aldehydes (notably 4-hydroxynonenal), and the end products malondialdehyde, ethane, and pentane. There are also markers of oxidative damage to proteins and DNA, namely protein carbonyls and 8-hydroxydeoxyguanosine respectively.

In 1989 , before the isolation of the cystic fibrosis gene, Salh et $a l^{6}$ suggested that aberrant free radical activity might underlie cellular dysfunction in the disease. Their evidence was based on measurement of the molar ratio of 
octadeca-9,11-dienoic acid to linoleic acid as an index of free radical attack on polyunsaturated fatty acids. They measured this marker in nasal epithelial cells and in serum and found it to be significantly elevated compared with values in appropriate controls. Measurements of serum levels of malondialdehyde were also made but were found not to be significantly increased in patients with cystic fibrosis. A similar observation on malondialdehyde had previously been made by Matkovics et $\mathrm{al}^{38}$; however, both investigations used thiobarbituric acid reactive substances (TBARS) as the marker of lipid peroxidation, and it is now recognised that these should not be used as the sole index. ${ }^{39}$ Reservations have also been expressed over the suitability of the molar ratio of octadeca-9,11-dienoic acid to linoleic acid as a marker, as the former may be produced by bacteria in the gut. ${ }^{40}$

In a recent study of cystic fibrosis patients attending an outpatient clinic at Southampton General Hospital ${ }^{41}$ the levels of two different markers of oxidative damage to lipids (TBARS and free fatty acid hydroperoxides), and of a marker of oxidative protein injury (protein carbonyls) were determined. Plasma concentrations of TBARS were significantly increased in patients compared with controls $(\mathrm{p}<0.05)$. This differs from the finding reported by Salh et $a l^{6}$ and may possibly be due to the fact that the control group in our study ${ }^{41}$ was age matched and had lower TBARS values than the adult control group in Salh's study (TBARS have been shown to increase with age ${ }^{42}$ ). Free fatty acid hydroperoxides, our second marker of lipid peroxidation, were not detectable in plasma from control patients which agrees with the results of Holley and Slater, ${ }^{43}$ but in 11 of 33 patients with cystic fibrosis they were present in a range of $0.022-0.34 \mu \mathrm{mol} / 1$. It therefore seems as if there is a subgroup of patients with cystic fibrosis who are susceptible to lipid peroxidation. There was no significant difference $(p=0.076)$ between the two groups for the marker of protein oxidation. Analysis of the major plasma antioxidants (ascorbic acid, $\alpha$-tocopherol, uric acid, and sulphydryls) revealed a relatively normal antioxidant profile except for uric acid which was decreased $(p<0.01)$ but still within the reported normal range. Thus, elevated indices of oxidative damage were detected in some patients with cystic fibrosis, even in the presence of relatively normal concentrations of antioxidants. These results suggest either that the plasma antioxidants cannot cope with the oxidative burden, or that the oxidative injury is occurring away from the plasma antioxidant pool - for example, intracellularly in the lung or extracellularly in the bronchioles - and that the products are transported into the plasma. From what is now known about the possible sources of oxidative stress in cystic fibrosis this would appear to be the case, but it is only the arrival of transgenic mouse models of cystic fibrosis that is allowing the more relevant issue of intracellular and bronchiolar antioxidant concentrations to be investigated.

\section{Future directions for research and clinical implications}

There appear to be two major sources of oxygen free radicals in cystic fibrosis: firstly, those produced by an underlying primary defect in intracellular oxygen metabolism, and secondly, those produced by neutrophils in the lung as a consequence of the pulmonary infection associated with the disease. At present it is not known which source, if any, dominates at any one time. It is possible, however, that the primary defect is responsible for a background level of radical production while episodic infectious periods result in heightened free radical activity. In recognising that free radical mediated damage may play a part in the pathogenesis of cystic fibrosis, it is important to address the clinical implication of these findings. For instance, there is evidence of increased oxidative damage to DNA in cystic fibrosis and the number of reports of cancers in patients with cystic fibrosis is rising as their life expectancy increases. ${ }^{4-47}$ The relation between the two may be exceedingly important, and may become more so with the advent of gene therapy. It is conceivable that, even if gene therapy should reduce the mortality of cystic fibrosis patients from pulmonary complications, their increased life expectancy may lead to an increase in malignancies due to an as yet unrecognised intracellular oxidative burden.

Patients with cystic fibrosis may benefit from an increased array of antioxidant defences against damage to macromolecules such as DNA, lipids, and proteins, as the evidence to date suggests that damage is occurring in spite of normal plasma antioxidant concentrations. This should prompt a review of what constitutes "adequate" antioxidant levels in the presence of a chronic oxidative burden, and we believe further investigation is warranted.

Until recently investigations along these lines have been extremely difficult, but with the development of transgenic mouse models of cystic fibrosis, tissue is becoming available and intracellular oxidant stress can be studied with greater precision. The mouse model also allows for more intense investigation of the pulmonary involvement of free radicals in the deterioration of lung function by focusing on the role of neutrophils and pulmonary antioxidants, where previously human bronchoalveolar lavage and biopsy samples were rarely available. The advent of genetic therapy for cystic fibrosis should not, however, be seen as a cure for this disabling disease, but as a spur to increased research into its pathology which may prove of great importance.

Ronan Brown is a Cystic Fibrosis Research Trust postgraduate student. These studies were supported, in part, by the Leopold Muller Trust.

Reprint requests to: Dr F J Kelly.

Cardiovascular Research,

The Rayne Institute,

St Thomas' Hospital,

London SE1 7EH,

$U K$

RONAN K BROWN FRANK J KELLY

1 Smith CV, Hansen TN, Martin NE, McMicken HW, Elliot SJ. Oxidant stress responses in premature infants during exposure to hyperoxia. Pediatr Res 1993;34:360-5.

2 Brigham KL. Oxidant stress and adult respiratory distress syndrome. Eur Respir f 1990;11(Suppl):482s-4s.

3 Owen S, Pearson D, Suarez-Mendez V, O'Driscoll R, Woodcock A. Evidence of free radical activity in asthma. N Engl f Med 1991;325:586-7.

Pryor WA. Ozone in all its reactive splendor. F Lab Clin Med 1993;122: Pryor WA. 6 .

5 Doring G, Albus A, Hoiby N. Immunological aspects of cystic fibrosis. Chest 1993;94:109S-15S

6 Salh B, Webb K, Guyan PM, Day JP, Wickens D, Griffin J, et al. Aberrant free radical activity in cystic fibrosis. Clin Chim Acta 1989;181:65-74.

7 Kneepkens CMF, Lepage G, Smith L, Lacaille F, Ronco N, Champagne J, et al. Low beta-carotene may contribute to the accumulation of free et al. Low beta-carotene may contribute to the a
radicals in cystic fibrosis. Pediatr Res 1991;29:106A.

8 Bye AME, Muller DPR, Wilson J, Wright VM, Mearns MB. Symptomatic vitamin E deficiency in cystic fibrosis. Arch Dis Child 1985;60:162-4.

9 Homnick DN, Cox JH, Deloof MJ, Ringer TV. Carotenoid levels in normal children and in children with cystic fibrosis. $\mathcal{F}$ Pediatr 1993;122:703-7.

10 Ater JL, Herbst JJ, Landaw SA, O'Brien RT. Relative anemia and iron deficiency in cystic fibrosis. Pediatrics 1983;71:810-4.

11 Hubbard VS, Barbero G, Chase HP. Selenium and cystic fibrosis. $f$ Pediatr 1980;96:421-2.

12 Stead RJ, Redington AN, Hinks LJ, Clayton BE, Hodson ME, Batten JC. Selenium deficiency and possible increased risk of carcinoma in adults with cystic fibrosis. Lancet 1985;ii:862-3.

13 Langley SC, Brown RK, Kelly FJ. Reduced free-radical-trapping capacity and altered plasma antioxidant status in cystic fibrosis. Pediatr Res 1993;33:247-50.

14 Migson FK, Koen R, Chevion M. Iron enhancement of ascorbate toxicity. Free Radic Res Commun 1989;5:107-15.

15 Langley SC, Brown RK, Kelly FJ. Extracellular antioxidant status in cystic fibrosis. In: Corongiu F, Banni S, Dessi MA, Rice-Evans C, eds. Free radicals and antioxidants in nutrition. London: Richelieu Press, 1993:13552 
16 Konstan $M W$, Berger $M$. Infection and inflammation of the lung in cystic fibrosis. In: Davis PB, ed. Cystic fibrosis. New York: Marcel Dekker, 1993:219-75.

17 Meyer KC, Zimmerman J. Neutrophil mediators, Pseudomonas, and pulmonary dysfunction in cystic fibrosis. $f$ Lab Clin Med 1993;121:654-61.

18 Graff I, Schram-Doumont A, Szpirer C. Defective protein kinase Cmediated actions in cystic fibrosis neutrophils. Cell Signal 1991;3:259-66.

19 Fick RB, Olchowski J, Squier SU, Merrill WW, Reynolds HY. Immunoglobin-G subclasses in cystic fibrosis. Am 7 Respir Dis 1986;133:418-22.

20 Meyer KC, Lewandoski JR, Zimmerman JJ, Nunley D, Calhoun WJ, Dopico GA. Human neutrophil elastase and elastase/alpha, -antiprotease complex in cystic fibrosis. Am F Respir Dis 1991;144:580-5.

21 Davis PB. Pathophysiology of the lung disease in cystic fibrosis. In: Davis PB, ed. Cystic fibrosis. New York: Marcel Dekker, 1993:193-218.

22 Reynolds HY, Newball HH. Analysis of proteins and respiratory cells obtained from human lungs by bronchoalveolar lavage. $7 \mathrm{Lab}$ Clin Med 1974;84:559-73.

23 Konstan MW, Norvell TM, Hilliard KA, Shiratsuchi H, Berger M. Serial bronchoalveolar lavage to evaluate inflammation in cystic fibrosis. Pediatr Pulmonol Suppl 1990;5:273.

24 Babior BM. The respiratory burst of phagocytes. $f$ Clin Invest 1984;73 599-601.

25 Weiss SJ. Tissue destruction by neutrophils. N Engl f Med 1989;320: 36576

26 Graft DF, Mischler E, Farrell PM. Granulocyte chemiluminescence in adolescent patients with cystic fibrosis. Am Rev Respir Dis 1982;125: 540-3.

27 Roberts RL, Stiehm RE. Increased phagocytic cell chemiluminescence in patients with cystic fibrosis. Am $\mathcal{F}$ Dis Child 1989;143:944-50.

28 Matthews WJ, Williams M, Oliphint B, Geha R, Colten HR. Hypogammaglobulinemia in patients with cystic fibrosis. $N$ Engl $f$ Med 1980;302:245-9.

29 Wisnieski JJ, Todd EW, Fuller RK, Jones PK, Dearborn DG, Boat TF, et al. Immune complexes and complement abnormalities in patients with cystic fibrosis: increased mortality associated with circulating immune complexes and decreased function of the alternative complement pathway. Am Rev Respir Dis 1985;132:770-6.

30 Britigan BE, Roeder TL, Rasmussen GT, Shasby DM, McCormick ML, Cox CD. Interaction of the Pseudomonas aeruginosa secretory products pyocyanin and pyochelin generates hydroxyl radical and causes synergistic damage to endothelial cells. F Clin Invest 1992;90:2187-96.

31 Coffman TJ, Cox CD, Edeker BL, Britigan BE. Possible role of bacterial siderophores in inflammation. Iron bound to the Pseudomonas siderophore pyochelin can function as a hydroxyl radical catalyst. $\mathcal{f}$ Clin Invest 1990;86:1030-7.
32 Kharazmi A, Rechnitzer C, Schiotz PO, Jensen T, Baek L, Høiby N. Priming of neutrophils for enhanced oxidative burst by sputum from cystic fibrosis patients with Pseudomonas aeruginosa infection. Eur $\mathcal{F}$ Biochem 1987;17:256-61

33 Feigal RJ, Shapiro BL. Mitochondrial calcium uptake and oxygen consumption in cystic fibrosis. Nature 1979;278:276-7.

34 Von Ruecker AA, Bertele R, Harms HK. Calcium metabolism and cystic fibrosis: mitochondrial abnormalities suggest a modification of the mitochondrial membrane. Pediatr Res 1984;18:594-9.

35 Turrens JF, Boveris A. Generation of superoxide anion by the NADH dehydrogenase of bovine heart mitochondria. Biochem $\mathcal{7}$ 1980;191:421-7.

36 Knoppert DC, Spino M, Beck R, Thiessen JJ, MacLeod S. Cystic fibrosis: enhanced theophylline metabolism may be linked to the disease. Clin Pharmacol Ther 1988;44:254-64.

37 Matkovics B, Gyurkovits K, Laszlo A, Szabo L. Altered SOD values in erythrocytes from children with Duchenne muscular dystrophy and cystic fibrosis. In: Greenwald RA, Cohen G, eds. Oxy radicals and their scavenger systems. Amsterdam: Elsevier, 1990:411-5.

38 Matkovics B, Gyurkovits K, Laszlo A, Szabo L. Altered peroxide metabolism in erythrocytes from children with cystic fibrosis. Clin Chim Acta 1982;125:59-62.

39 Draper HH, Squires EJ, Mahmoodi H, Agarwal S, Wu J, Hadley M. A comparative evaluation of thiobarbituric acid methods for the determination of malondialdehyde in biological materials. Free Radic Biol Med 1993;15:353-63.

40 Wickens DG, Dormandy TL. The possible origins of human octadeca9,11-dienoic acid. In: Rice-Evans C, Dormandy TL, eds. Free radicals: chemistry, pathology and medicine. London: Richelieu Press, 1988:237-2.

41 Brown RK, Kelly FJ. Evidence for increased oxidative damage in patients with cystic fibrosis (CF). Pediatr Res 1994;35 (in press).

42 Yagi K. Assay for blood plasma or serum. In: Packer L, ed. Methods in enzymology. London: Academic Press, 1984:328-31.

43 Holley AE, Slater TF. Measurement of lipid hydroperoxides in normal human blood plasma using HPLC-chemiluminescence linked to a diode array detector for measuring conjugated dienes. Free Radic Res Commun 1991;15:51-63.

44 Davis TM, Sawicka EH. Adenocarcinoma in cystic fibrosis. Thorax 1985;40:199-200.

45 Biggs BG, Vaughan W, Colombo JL, Sanger W, Purtilo DT. Cystic fibrosis complicated by acute leukemia. Cancer 1986;57:2441-3.

46 Tedesco FJ, Brown R, Schuman BM. Pancreatic carcinoma in a patient with cystic fibrosis. Gastrointest Endosc 1986;32:25-6.

47 Neglia JP, Wielinski CL, Warwick WJ. Cancer risk among patients with cystic fibrosis. $\mathcal{F}$ Pediatr 1991;119:764-7. 